\title{
A Global Architecture for a Quality of Service Model for Ad Hoc Networks
}

\author{
Hassene Bouhouch \\ SecNum Laboratory Research, SUP'COM \\ High School of Communication SUP'COM, \\ University of the 7th of November at Carthage, Tunisia
}

\author{
Sihem Guemara El Fatmi \\ SecNum Laboratory Research, SUP'COM \\ High School of Communication SUP'COM, \\ University of the 7th of November at Carthage, Tunisia
}

\begin{abstract}
Ad Hoc mobile Network (AHNs) may include a plurality of mobile nodes each including a wireless communications device with limited communication resources. Achieving QoS (Quality of Service) in AHNs has been an important research topic in the last years. Hence, we are interested in this paper by the proposition of a global architecture for the offer of a QoS model for AHNs users.

Our proposition is based on traffic classification and requires metrics definition allowing the expression of the QoS requirements in order to provide differentiated treatment according to traffic classes. One of the major contributions of this paper concerns the proposition of a novel QoS routing protocol based on delay and bandwidth requirements and called QSDB. Then, we are interested by the proposition of a novel contention resolution mechanism allowing an admission control oriented service differentiation mechanism called DS_CAP. Finally, we propose a QoS reservation mechanism for AHNs, called QSRR. The mechanism uses a traffic classification and requires available bandwidth estimation definition. The advantages of our propositions are shown thanks to some simulation results that are detailed in the end of this paper.
\end{abstract}

\section{General Terms}

routing protocol, admission control, collision management, resources reservation mechanism, quality of service, Ad Hoc networks (MANET's)

\section{Keywords}

QoS routing protocol, QoS parameters, Admission control, QoS resources reservation mechanism, Ad Hoc networks.

\section{INTRODUCTION}

Today, the rise of wireless technologies offers new prospects in the telecommunications field. The recent evolution of wireless communication means allows the handling of information through portable units with particular characteristics such that modest flows and communication resources, autonomous and limited source of energy and frequent disconnections. Wireless networks can be classified into two categories: networks with infrastructure such as found at the cellular technology and networks without infrastructure or Ad Hoc networks. The absence of infrastructure in the second category leads some problems. In particular, in order to ensure the transmission of information through the network, the mobile node performs the role of a station and a router. Moreover, the radio link specificities as well as the mobility of the users make unacceptable the routing protocols used in the usual networks. Because the QoS provision constitutes a real challenge for current networks and because this aspect has not been sufficiently dealt with in AHNs, we have chosen to focus our attention on this issue by the proposition of a global architecture for a QoS model for the AHN users. This architecture provide a QoS routing protocol, a differentiated service medium access control protocol and a resources reservation mechanism satisfying QoS constraints. Particularly, this paper aims first to propose a routing protocol in AHNs QoS oriented i.e. allowing customized treatments for the different traffics according to their priorities. In a routing protocol QoS oriented, the paths are selected in agreement with QoS required by the applications. In a second stage, the reader will find in this paper a collision management mechanism and an admission control oriented QoS which will be adopted for introducing services differentiation for the AHNs user. The third contribution of this paper concerns the introduction of a novel proposition of resources reservation mechanism that takes into account the QoS challenge.

This paper is organized as follows: the $\mathrm{Ad} \mathrm{Hoc} \mathrm{mobile}$ environment's is very particular and the AHNs unit's constraints are very strong, we think it right to study in the second section of this paper these particularities. This section also deals with the concept of QoS in AHNs and details the classes of traffic (Real Time/ Best Effort). The third section defines the global architecture of a QoS model for AHNs. In section 4, we introduce the QoS routing protocol, called QSDB (Quality of Service Delay and Bandwidth Based) so that the network can offer QoS to its users. We have to differentiate the treatment to be adopted according to the type of traffic by creating various routing tables sensitive to different metrics (delay, throughput, maximum available bandwidth) to offer to each type of application its own type of "optimal path". Section 5 defines the functioning of the admission control mechanism and presents the collision management mechanism and the admission control oriented QoS, called DS_CAP (Differentiation Service Control Admission Protocol), which will be adopted for introducing services differentiation for the AHNs users. The sixth section of this paper exposes a novel proposition of resources reservation mechanism, called QSRR that takes into account the QoS challenge by defining a relation between the required constraints of the applications. In section 7, we expose the simulation results. Section 8 concludes this paper.

\section{Quality OF Service In AD Hoc NETWORKS}

In this section we study the Ad Hoc networks environment's and the mobile unit's characteristics, the concepts of QoS in AHNS and finally we details the classes of traffic.

\subsection{The Ad Hoc Networks characteristics}

AHNs started with the aim to have the ability to establish a network among willing nodes without the assistance from any network infrastructure. AHNs are defined as a 'collection of mobile entities interconnected by a wireless technology forming a temporary network without the assistance of any administration or any fixed support where no centralized administration is available' [1]. Contrary to a cellular network, they are the mobile hosts themselves which form, in an ad hoc way, the network infrastructure. This ability to establish this type of network seems 
to be very promising in situations like disaster or war (where the infrastructure is damaged or not available) or in areas where building the infrastructure is not possible or situations where an AHN is more suitable than an infrastructure network.

AHNs, which are based on IEEE 802.11 standard, are characterized by several limits and constraints [2] such as limited and shared bandwidth, limited energy, poor security, and so on. These characteristics make big differences between AHNs functioning and the one of traditional wired networks. In fact, dynamic topology and nodes arbitrary mobility can aggravate these difficulties especially because of the fast movements of the nodes and the variable conditions of the network which make the information of the network's state, obsolete quickly. Because of these characteristics, AHNs suffer of several problems such as the routing, the management of the mobility, the security, the quality of service and essentially what most interests us, the resources reservation. In fact, the resources reservation process constitutes one of the more illustrative and the more heavy examples of such differences between AHNs functioning and the one of traditional wired networks.

\subsection{Quality of Service in Ad Hoc Networks}

The QoS provided by a network constitutes nowadays an important issue for advanced applications because it concerns the different needs and constraints characterizing these applications. From the part of the network, it indicates its capacity to transport, under good conditions, flows resulting from various applications and various users [3]. Generally, it expresses a measure of the level of service that a particular data gets in the network. The basic idea behind 'provided' service is to differentiate between traffic coming into the network and provide preferential treatment to some types of data (Real Time applications). QoS also expresses a measure of performance and/or user satisfaction relative to a transmission system that reflects its transmission quality and availability of service. The QoS can be defined as the manner that the service of delivery of packages is supplied and who can be characterized by various parameters of performance like the availability, the rate of errors, the response time, the delay, the throughput, the delay variation (jitter), the packet loss etc [4].

We believe for AHNs, with time-varying low-capacity resources, the notion of being able to guarantee hard QoS is not plausible. Instead, applications must adapt to time-varying low-capacity resources offered by the network. Therefore, the QoS that an application requires depends on the "quality" of the network. This "quality" should be a function of available resources resides both in the wireless medium and in the mobile nodes in the network as well as the stability of such resources. Hence, QoS in AHNs could mean to provide a set of parameters in order to adapt the applications to the "quality" of network while routing them through the network. Providing QoS in AHNs has its own challenges and problems. For the QoS in the AHNs, some major networks aspects characterize a QoS expressed by a user or delivered by a network [5]. They are (1) the delay, (2) the resource availability and/or capacity, (3) the reliability, the bandwidth (4) and the rate of errors (5). We still speak about 'Capacity of the network'.

\subsection{Traffic classes}

We have considered three classes of traffic according to the applications QoS requirements. One on these classes has no QoS constraints [6]. The two others have strong temporal constraints and one of them has in addition strong bandwidth constraints. The three classes can be detailed as follows:

- Class 0: Real Time traffic Delay sensitive (CBR) generated by applications having strong temporal constraints: each bandwidth allowing the requested delay is acceptable by such traffic class.
- Class 1: Real Time traffic Bandwidth sensitive (VBR_RT) generated by applications having strong temporal constraints in addition to strong constraints in terms of bandwidth.

- Class 2: Best Effort Traffic (BE) generated by applications having no QoS constraints.

\section{Global Architecture For The QOS MODEL}

The most used design for AHN architecture is the 'cross layer' design which differ from the traditional ones where each layer operate independently. This architecture try to improve the performances of the system by implementing several connected protocols layers. It so allows the higher layers to adapt their strategies to the variations of the links and in function to the conditions of the network. Such design gives the possibility to the layers to interact together. Each layer is characterized by some key parameters which are passed to the others layers allowing them to determine the operational mode which answers at best at the same moment in the conditions of the network, of the channel and of the applications. Layers are not dependent the some in the others, but they cooperate between them to find the optimal behaviour to adopt.

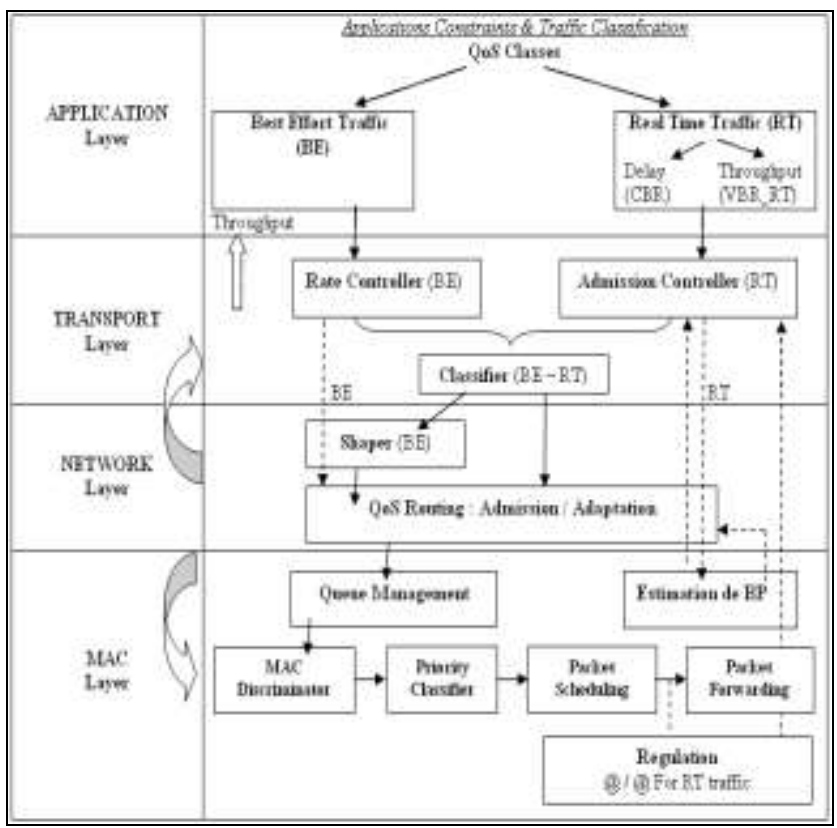

Figure 1. Global Architecture for the QoS model.

The proposed QoS model, illustrated in Figure 1, take into account the AHN constraints such as limited transmission rate, the paths changing, the packets loss, the nodes mobility, etc. To provide an appropriate QoS for the transmitted applications, it is necessary to set up a lot of protocols and mechanisms working according to the nature of the applications:

- A QoS oriented routing protocol,

- Admission control mechanism according to the applications requirements in term of QoS, the establishment of the path (in association with the QoS routing protocol) and the establishment of the control messages to set up the parameters.

- Resources allocation mechanism.

\section{QOS BASEd Routing AT Ad HoC NETWORKS}

In AHNs, the routing has to guarantee, at any moment, connection between any pair of nodes belonging to the network whatever the states and the positions of the mobile units. It has to 
take into account all changes occurring in the network such as topology, bandwidth, number of links, network resources and so on. Each node insures the role of station and router and takes part in the routing protocol to discover the paths to reach the other nodes. These characteristics make very difficult to determine and particularly to preserve the optimal path to reach the destination node.

\subsection{A QoS routing protocol proposition: QSDB}

The routing protocol that we propose takes into account a large scale of QoS parameters to allow application to clearly specify its requirements. The selected path must assure an average delay and an average throughput to respect the different exigencies of the various traffic classes, especially the Real Time traffic. This protocol is called QSDB, "Quality of Service Delay and Bandwidth Based Routing Protocol" [7].

For the path selection, the nodes must declare their characteristics to allow the choosing of the best rode in term of delay and available bandwidth. The available bandwidth on a link can be defined as the maximum throughput that can be transmitted between two peers without disrupting any ongoing flow in the network. This term should not be confused with the link capacity that represents the maximum throughput a flow can achieve between two nodes. For the CBR applications (delay sensitive), the routing protocol must offer paths having the best Delay satisfying, as in the relation (1):

DELAY MIN < = DELAY (path) < = MAPD (1)

Where: Delay MIN: the minimal delay required, and, MAPD: Maximum Acceptable Packet Delay.

For the VBR_RT applications (throughput sensitive), the routing protocol must offer paths having bandwidth larger than the minimal bandwidth required by the application, as in the relation (2).

$$
\text { MAB (path) >= Bandwidth MIN required }
$$

The routing protocol must be able to collect some information about these metrics: delay, available bandwidth and maximum throughput. In fact, each node transmits three signalling messages:

- LDA (Local Delay Advertisement) that contains necessary delay to join all direct neighbors of the concerned node $\rightarrow$ Delays declaration.

- RBA (Remaining Bandwidth Advertisement) that contains the available bandwidth on links relaying this node to all its direct neighbors' $\rightarrow$ Available bandwidth quantities declaration.

- HELLO that contains all information about neighbors and their link's state $\rightarrow$ Neighbors Declaration.

In this manner, we have in each node a state table containing neighbour address (thanks to HELLO message), necessary delay to join it (thanks to LDA message) and also available bandwidth on this link between these two nodes (thanks to RBA message). For a path containing $\mathrm{n}$ nodes, we have:

- The Delay can be expressed as follows:

Let assume that $D(i, j)$ is the link $(i, j)$ delay haven thanks to the LDA message; For a path $\mathrm{p}=(\mathrm{S}, \mathrm{i}, \mathrm{j}, \ldots, \mathrm{D})$; where $\mathrm{S}$ : Source, $\mathrm{D}$ : Destination and $\mathrm{i}, \mathrm{j}, \ldots$ intermediary nodes, we have as expressed in (3) :

$$
\mathbf{D}(\mathbf{p})=\mathbf{D}(\mathbf{S}, \mathbf{i})+\mathbf{D}(\mathbf{i}, \mathbf{j})+\ldots \ldots+\mathbf{D}(\mathbf{k}, \mathbf{D})
$$

- The Average Bandwidth can be expressed as follows:

Let assume that $\mathrm{AB}(\mathrm{i}, \mathrm{j})$ is the available bandwidth on the link $(i, j)$ haven thanks to the RBA message; For a path $\mathrm{p}=(\mathrm{S}, \mathrm{i}, \mathrm{j}, \ldots$, D); we have as expressed in formula (4):

$$
A B(p)=A B(S, i)+A B(i, j)+\ldots \ldots . .+A B(k, D)
$$

- The average bandwidth can be expressed as follows in (5):

$$
\text { Average Bandwidth }=\mathbf{A B}(\mathbf{p}) / \mathbf{n}
$$

where $\mathrm{n}$ is the link number on the path S-D.

- The Maximum Average Bandwidth (MAB) can be expressed as follows in formula (6):

$\operatorname{MAB}(p)=\operatorname{MIN}\{\operatorname{MAB}(S, i), \operatorname{MAB}(i, j), \ldots, \operatorname{MAB}(k, D)\}(6)$ Let assume that $\operatorname{MAB}(i, j)$ is the maximum available bandwidth on the link (i, j) (defined later in the function (7)) and $\mathrm{p}=(\mathrm{S}, \mathrm{i}$, $\mathrm{j}$...D) is a path.

\subsection{Available bandwidth prevision}

In AHNs, available bandwidth in each node depends both of his consumption, the consumption of all his direct neighbors and the interferences caused by all these transmissions. Otherwise, one application can not optimize its transmission without having a precise idea of the complete state of his neighborhoods in term of resources consumption. The available bandwidth estimation is a fundamental operation for a QoS offer [8]. This operation is very difficult because of the approximate acknowledge of the network state and the random mobility of nodes.

For AHNs, this mechanism is generally placed in the MAC layer to permit source to estimate the available bandwidth quantities. This estimation must to take into account node's mobility, interferences caused by the different transmissions and the hidden stations problem. It must be permanently up to date especially after a congestion establishment or a reception of a duplicate ack.

Available bandwidth can be defined as the maximum throughput with which we can transmit (between two nodes) without interrupt flows transmitted on the networks. This term must not to be confused with the 'link capacity' representing the maximum throughput which can attempt on this link, or with the 'unusable link capacity'. Knowledge of the available bandwidth quantity is required for admission control, bandwidth based routing and flow management [9]. Quantitatively, this available quantity can be expressed as follows:

- Let assume that BW is the total bandwidth quantity on a node.

- The maximum available bandwidth quantity on a node can be defined by this function (7):

Where:

$$
\operatorname{MAB}(\mathbf{i})=\mathbf{B W}(\mathbf{i})-\mathbf{x}(\mathbf{i})-\mathrm{SUM}_{\mathbf{j} \epsilon \mathrm{Ni}} \mathbf{x}(\mathbf{j})
$$

- $\quad$ BW (i) $=$ Total bandwidth on the node $i$,

- $\quad x(i)=$ Used Bandwidth on the node $i$,

- $\quad x(j)=$ Used Bandwidth by the node $j$ neighbor of the node $\mathrm{i}$,

- Node $\mathrm{j}=$ neighbor of node $\mathrm{i}$, and,

- $\mathrm{Ni}=$ set of node $\mathrm{i}$.

- On a link $(i, j)$, the available bandwidth is already expressed as follows in (8):

$$
\mathrm{AB}(\mathbf{i}, \mathbf{j})=\operatorname{MIN}\{\operatorname{MAB}(\mathbf{i}), \operatorname{MAB}(\mathbf{j})\}
$$

\section{COLLiSion MANAgEMENT AND ADMisSion CONTROL ORIENTED SERVICE DifFERENTIATION PROPOSITION}

The aim of the mechanism presented in this section is to provide a service differentiation using the MAC 802.11 layer parameters and system priorities. The service differentiation on the MAC layer is made according various parameters of DCF function to introduce priorities between data flows and it is based on the contention window size $(\mathrm{CW})$ of the Backoff algorithm. By proceeding to a differentiation of service at the MAC layer, we can offer to the applications, different priorities of access to the medium. This access, following the DCF and PCF functions of the IEEE 802.11 standard, can be summarized in these points: 
- The utilization of the CSMA access method to be able to avoid the collisions between the various data flows.

- The utilization of the signaling message RTS/CTS which allow the control of the access to the radio medium as well as the resolution of the problem of the hidden stations.

- The utilization of the control message ACK allowing the source the possibility of starting a retransmission if the data packet did not arrive at destination or if it arrived with a bad code CRC (corrupted).

- The utilization of the Backoff algorithm whose role is the limitation of the collisions by proceeding to a random waiting delay before the transmission of a flow of data.

\subsection{Backoff service differentiation based}

The aim of this section is to propose a modification of the Backoff algorithm that considers various QoS classes. Let's first recall that the original Backoff algorithm [10] calculates, as expressed in formula 9, the waiting delay before a transmission attempt according to the following function:

T Backoff $=\operatorname{random}\left(0,2^{(i-1)} * \mathbf{C W}\right) *$ Timeslot

Where $\mathrm{i}$ is the attempt number, $\mathrm{CW}$ is the contention window size computed according to the function (10).

$$
\text { CWnew }=\min (\text { CWmax, CWold } * 2)
$$

For the three traffic classes considered in this paper, only the Backoff expression of Real Time traffic (class 0 and 1) is modified. The distinction made between a Best Effort traffic and real time traffic constitutes the major basis for the service differentiation at this level. For these traffic classes, we offer a differentiated service to high priority classes. We have added to the initial formula a differentiation relative to the traffic class $(0$ or 1) by the introduction of a variable persistent factor (PF) and a variable maximum size of $\mathrm{CW}$. This variability appears in the following expression (11):

CWnew [i] $=\min ($ CWmax [i]; CWold [i] * PF [i] $)$ where i represent the traffic class $(0$ or 1$)$.

In conclusion, we can notice that a service differentiation has been made in two levels. First, between Best Effort traffic and Real Time traffic and second, between class 0 and class 1 for the Real Time traffic. The differentiation has been made using variability into contention window size and persistent factor.

During the rest of this section, we are going to focus on the service differentiation at the MAC layer and the traffic classes' differentiation which allows us to provide a complete admission control oriented service differentiation mechanism. These mechanisms are the concepts of our DS_CAP proposition, Differentiated Service Control Access Protocol [11]

\subsection{Service differentiation at the MAC layer}

The service differentiation introduction in IEEE 802.11 and the service differentiation between traffic classes that we propose in this section is made using:

- Various timers (PIFS / DIFS) ensuring that there is no high waiting priority packet when a low priority packet is transmitted. In this way, the Best Effort applications will use a DIFS delay (Distributed Inter Frame Space) relative to the DCF function and the Real Time applications will use a PIFS delay (Point Coordination Function Inter Frame Space) relative to the PCF function. Naturally, the PIFS delay is shorter than the DIFS one so that the Real Time applications will always have the priority with regard to the Best Effort applications.

- Differentiated Backoff Algorithm ensuring that each traffic class has its own Backoff algorithm.

CWnew [i] $=\min ($ CWmax [i] ; CWold [i] * PF [i] $)(11)$ Besides, we have the possibility of making a differentiation on PF [i], CWmin [i] and CWmax [i].
The remaining part of this section is to detail this service differentiation according the traffic class. The differentiation of the services at the MAC layer consists in playing on the various parameters of the DCF function used by the method of access CSMA/CA to introduce priorities between the various flows of data. These various parameters can be the following ones:

- A SIFS delay which varying with the priority level of the applications.

- A DIFS delay which varying with the priority level of the applications. It is interesting to choice a DIFS delay for the Best Effort applications and a PIFS delay for the Real Time applications.

- Limit the waiting delay before the retransmission after the collision detection according to the priority level of the applications using the differentiated Backoff algorithm.

\subsection{Traffic classes differentiation}

In this subsection, we suggest differentiating the policy of access to the channel according to the nature of flows.

Classes 0 and 1 service

- The use of a unique RTS/CTS request sent at the beginning of transmission. In fact, in order to advantage such traffic, this signalling is valid for several transmissions and it has to be reinitialized only an ACK is lost or the maximum delay of transmission is reached.

- A choice for a short delay PIFS relative to the PCF function.

- Utilization of the modified Backoff algorithm where the size of the Contention Window $(\mathrm{CW})$ increases but not doubles on each new retransmission tentative.

CWnew $[i]=\min ($ CWmax $[i] ;$ CWold [i] * PF [i] $)$

Class 2 service

- A RTS/CTS request sent before each transmission. This signalling has to be renewed for each new packet.

- A choice for a DIFS delay can be tolerable.

- Utilization of a classic Backoff algorithm where the size of the $\mathrm{CW}$ double on each new retransmission tentative:

CWnew $=\min [\mathrm{CWmax} ; 2 *$ CWold $]$

\section{RESOURCES RESERVATION MECHANISM}

AHNs resources reservation is a challenging task due to the lack of resources both in the wireless medium and in the mobile nodes as well as the frequent changes in network topology. As a result, resources reservation in such networks is more difficult than in wired networks. Moreover, in AHNs, it is essential to consider the quality of links and to take into account the time-varying topology and time-varying network resources.

An important problem associated with resources reservation in AHNs is to employ methods that ensure the adequate QoS for the applications. The running of a service through an AHN will be interrupted, if an intermediate node belonging to the path moves out of range during data transfers. This interruption requires a subsequent path re-discovery between the source and the destination and invokes some path-maintenance algorithm that eventually increases the end-to-end delay. For instance, it is possible that a path that was earlier found to satisfy some QoS requirements no longer does so due to the dynamic nature of the topology. In such case, it is important that the network intelligently adapts the session to its new and changed conditions. The goal of our QoS resources reservation method, QSRR [12], is two-folds: first reserving network path that have sufficient resources to satisfy the QoS requirements of all admitted connections and second achieving global efficiency in resource utilization. For each flow on which we have provided some QoS guarantee, QSRR will allocate some resources which will be exclusively for its use. This will ensure that as soon as the packet 
of that particular flow comes, it will not have to wait for some path or resource to be freed and it will be transmitted to the next node instantly.

During the rest of this section, we will interest to the expression of a relation between the required constraints in term of delay and bandwidth in order to express the applications requirements.

\subsection{Delay and Bandwidth Computing}

QoS-adaptation provides an interface for applications to submit their requirements. Some applications are capable to expand their QoS parameters, so that instead of being a single value indicating the constraints (in term of delay or throughput) needed by an application; it becomes a range of service classes in which the application can operate, together with the current reserved value within that range. This provides the network flexibility so that reservations can be maintained as network conditions change. Applications request QoS by specifying the minimum level of service they are willing to accept and the maximum level of service they are able to utilize, and then adapt to the specified point within this range that the network commits to provide, which may change with time. Changes in allocation have to be signalled to the application, which adapts its behaviour to match to what is available.

To offer bandwidth guaranteed QoS, the residual end-to-end bandwidth must be known. In wired networks this is a trivial task since the underlying medium is a dedicated point-to point link with fixed capability. However, in wireless networks the radio channel of every node is shared with all its neighbours. Due to the shared medium, a node can successfully use the channel only when all its neighbours do not transmit and receive packets simultaneously. While the resources reservation is based on the available bandwidth, we use, in QSRR, an efficient method to obtain a relation between the required delay and the required bandwidth, as expressed in formula (12) and (13):

$$
\begin{aligned}
D \text { req } & =\frac{B \text { req }}{B \text { max }-(B \text { res }+ \text { B req })} \\
\text { B req }= & \frac{D \text { req }(B \text { max }- \text { B res })}{1+D \text { req }}
\end{aligned}
$$

Where:

- $\mathrm{D}$ req: is the requested delay;

- $B$ req: is the requested bandwidth;

- B max: is the maximum bandwidth supported by a link;

- $B$ res: is the residual bandwidth.

\section{SimUlation RESULTS}

Once the proposed QoS protocols and mechanisms concepts have been completely defined, it becomes necessary to check their feasibility and evaluate their benefits. Simulation analysis is used here to evaluate the proposed scheme. The goal of this section is to present the main aspects of the simulation model and its results.

\subsection{Simulations Environnement}

Two categories of parameters have been considered in our study: the input parameters and the output parameters. These parameters are as follows:

Input parameters: These include:

Tow types of applications: CBR \& VBR_RT.

Tow types QoS oriented Routing protocol: OLSR (Optimized Link State Routing) and our protocol QSDB (Quality of Service Delay and Bandwidth Based).
- Tow types QoS oriented MAC protocol: CSMA and our medium access control protocol, DS_CAP.

- Tow types of Resources Reservation Mechanism: CSMA and our resources reservation mechanism, QSRR.

- A number of nodes constituting our network: variable between 10 and 50 (interval of 5 nodes).

Output parameters: These include:

Delay (sec),

- Throughput (packets/sec),

- Delivery Rate $(\%)=\frac{\text { Routing Traffic Received }}{\text { Routing Traffic Sent }}$
- $\quad$ Media Access Delay (sec), and,
- Load (bps)

\subsection{Simulations Scenarios}

For the applications type, we use:

- Only CBR applications, or,

- Only VBR_RT applications, or,

- Both CBR and VBR_RT applications.

For the routing protocol, we use:

- OLSR routing protocol, or,

- QSDB routing protocol.

For the Medium Admission Control protocol, we use:

- CSMA protocol, or,

- Our DS_CAP protocol.

For the Resources Reservation Mechanism, we use:

- CSMA protocol, or,

- Our QSRR mechanism.

The node number is varying between 10 and 50 .

\subsection{Simulations Results}

\subsubsection{Simulation results for QSDB routing protocol}

In this sub-section, we try to show that the performances provided by our protocol, QSDB, are always better than the ones provided by the OLSR protocol. Theses performances are expressed in terms of delay, throughput and delivery rate.

\subsubsection{Protocols comparison in terms of delay}

In a general manner, we notice that the delay increases with the increase of the number of nodes constituting the network, but the delay provided by our protocol QSDB remains always lower than the one provided by OLSR and this whatever the distribution of the applications (\% of CBR applications and \% of VBR_RT applications) and whatever the number of nodes constituting our network. The Figure.2, Figure.3 and Figure.4 represent the QSDB delay's evolution and OLSR delay's evolution respectively for the application distribution 100\%CBR and 0\%VBR_RT, 50\%CBR and 50\%VBR_RT and finally 0\%CBR and $100 \%$ VBR_RT.

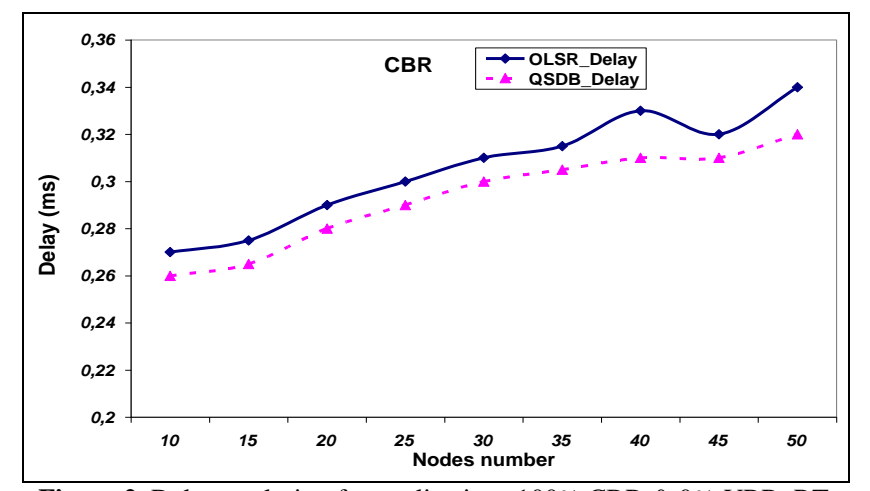

Figure 2. Delay evolution for applications 100\% CBR \& 0\% VBR_RT 


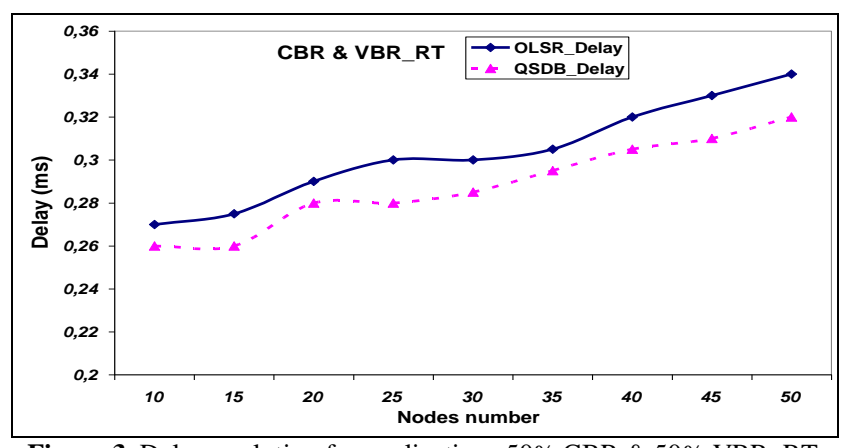

Figure 3. Delay evolution for applications 50\% CBR \& 50\% VBR_RT

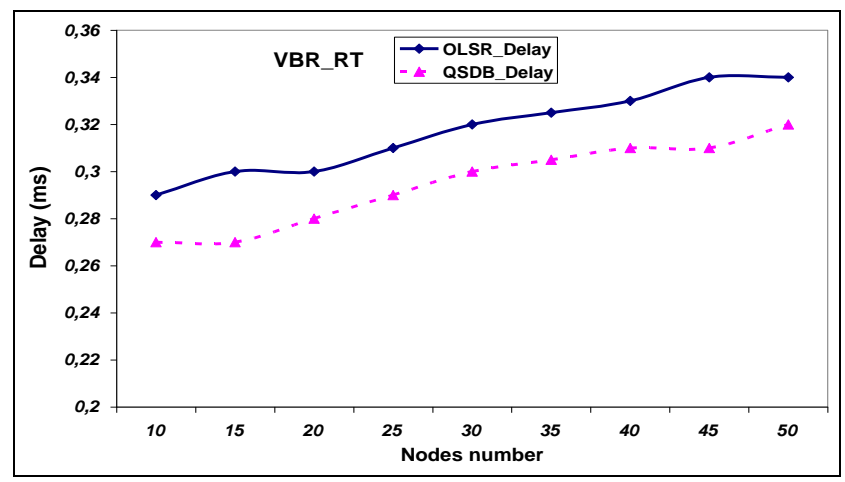

Figure 4. Delay evolution for applications 0\% CBR \& 100\% VBR_RT

\subsubsection{Protocols comparison in terms of delivery rate}

In a general manner, we notice that the delivery rate decreases with the increase of the number of nodes constituting the network, but the delivery rate produced by QSDB remains always upper to that the one produced by OLSR, and this whatever the application's distribution and whatever the number of nodes constituting the network.

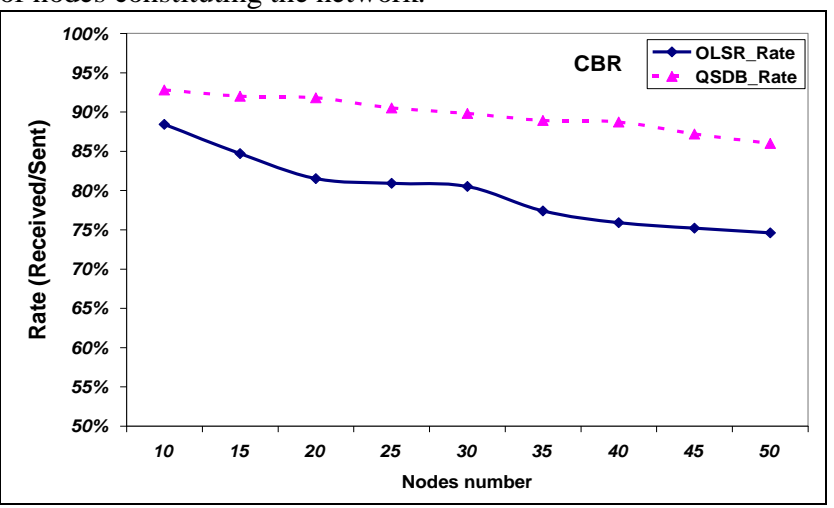

Figure 5. Delivery rate evolution for applications $100 \%$ CBR \& 0\% VBR_RT

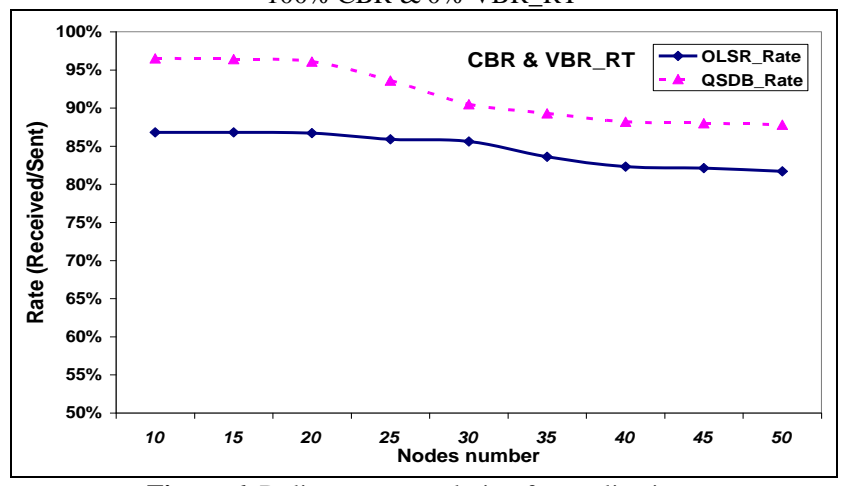

Figure 6. Delivery rate evolution for applications 0\%CBR\&50\%VBR_RT

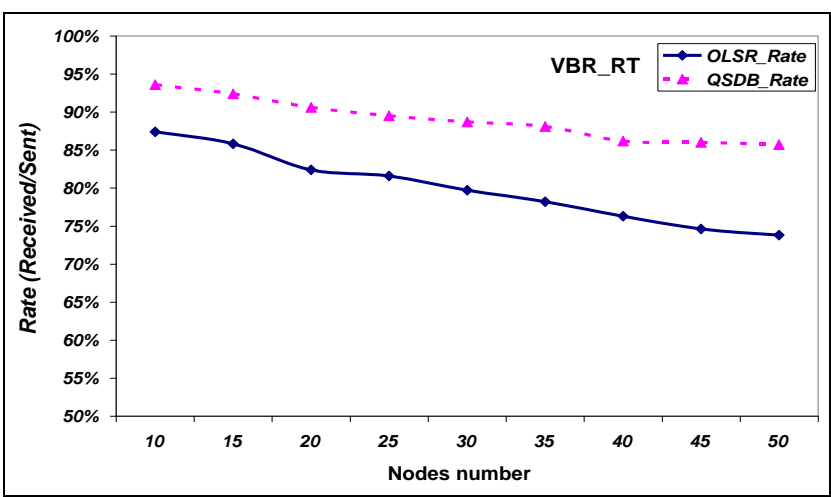

Figure 7. Delivery rate evolution for applications 0\%CBR\&100\%VBR_RT

The Figure.5, Figure.6 and Figure.7 represent the QSDB delivery rate's evolution and OLSR delivery rate's evolution respectively for the application distribution 100\%CBR and 0\%VBR_RT, 50\%CBR and 50\%VBR_RT and finally 0\%CBR and $100 \%$ VBR_RT.

\subsubsection{Protocols comparison in terms of Throughput}

In a general manner, we notice that the throughput increases with the increase of the number of nodes constituting the network, but the throughput provided by QSDB remains always upper to that the one provided by OLSR, and this whatever the application's distribution and whatever the number of nodes constituting the network. The Figure.8, Figure.9 and Figure.10 represent the QSDB throughput's evolution and OLSR throughput's evolution respectively for the application distribution 100\%CBR and 0\%VBR_RT, 50\%CBR and 50\%VBR_RT and finally 0\%CBR and $100 \%$ VBR_RT.

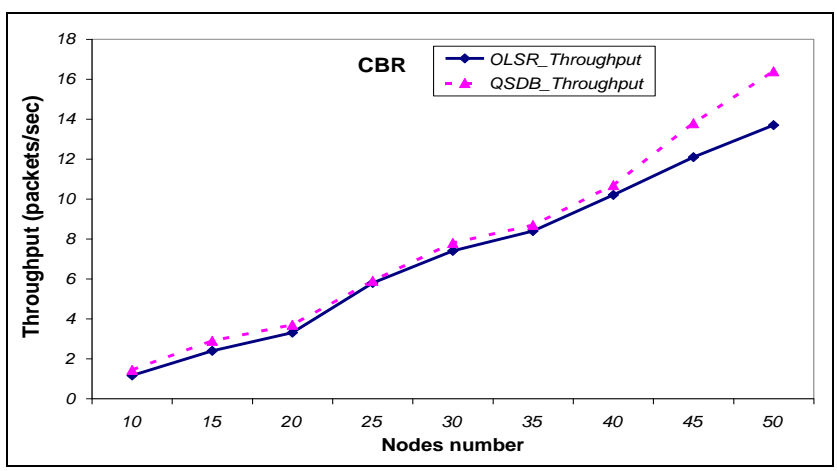

Figure 8. Throughput evolution for applications $100 \%$ CBR\&0\%VBR_RT

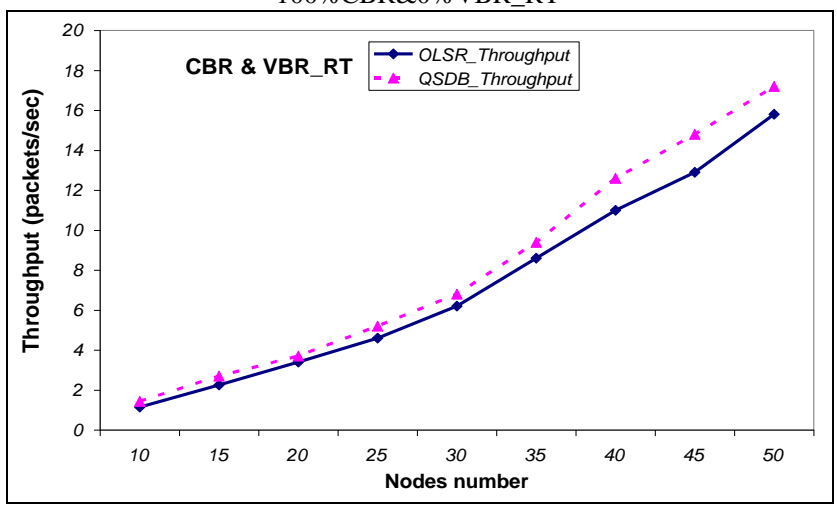

Figure 9. Throughput evolution for applications $50 \%$ CBR\&50\%VBR_RT 


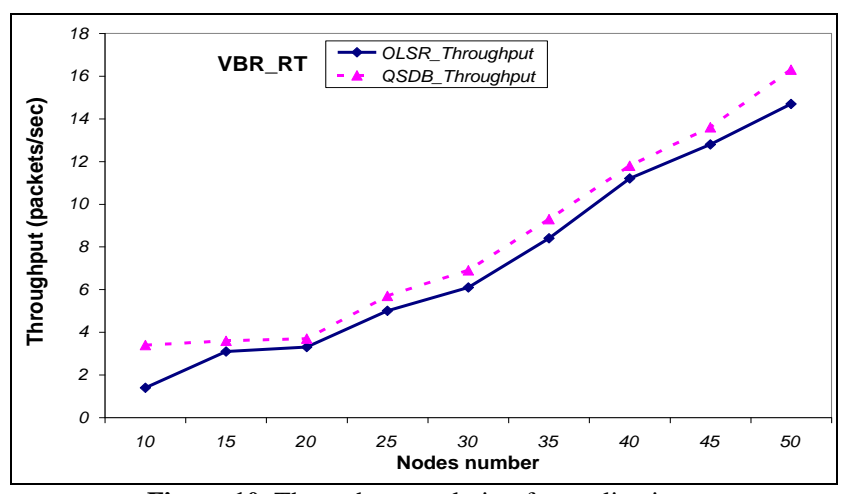

Figure 10. Throughput evolution for applications 0\%CBR\&100\%VBR_RT

\subsubsection{Simulation results for DS_CAP protocol}

In this sub-section, we try to show that the performances provided by our protocol, DS_CAP, are always better than the ones provided by CSMA. Theses performances are expressed in terms on media access delay.

In a general manner, we notice that the media access delay increases with the increase of the number of nodes in the network, but the media access delay provided by DS_CAP remains always lower than the one provided by CSMA, and this whatever the distribution of the applications and whatever the number of nodes constituting our network. The Figure.11, Figure.12 and Figure.13 represent the DS_CAP media access delay's evolution and CSMA media access delay's evolution respectively for the application distribution 100\%CBR and 0\%VBR_RT, 50\%CBR and 50\%VBR_RT and finally 0\%CBR and $100 \%$ VBR_RT.

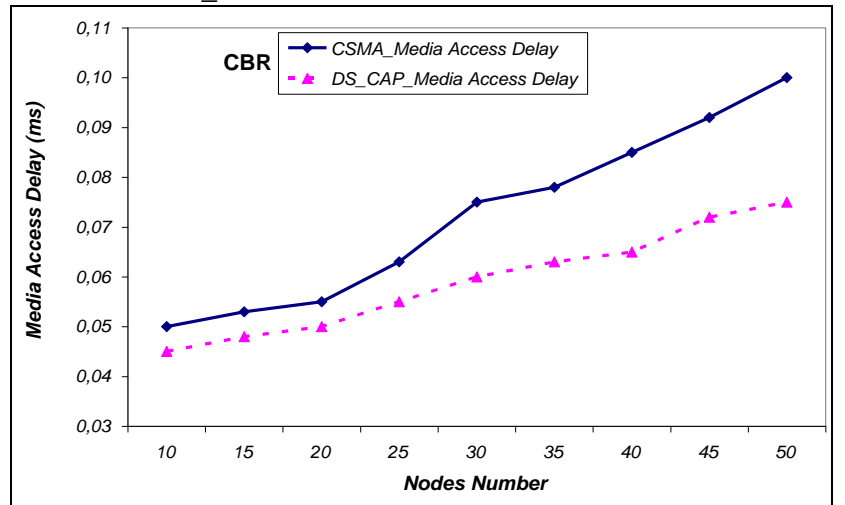

Figure 11. Media Access Delay evolution for applications $100 \%$ CBR \& $0 \%$ VBR RT

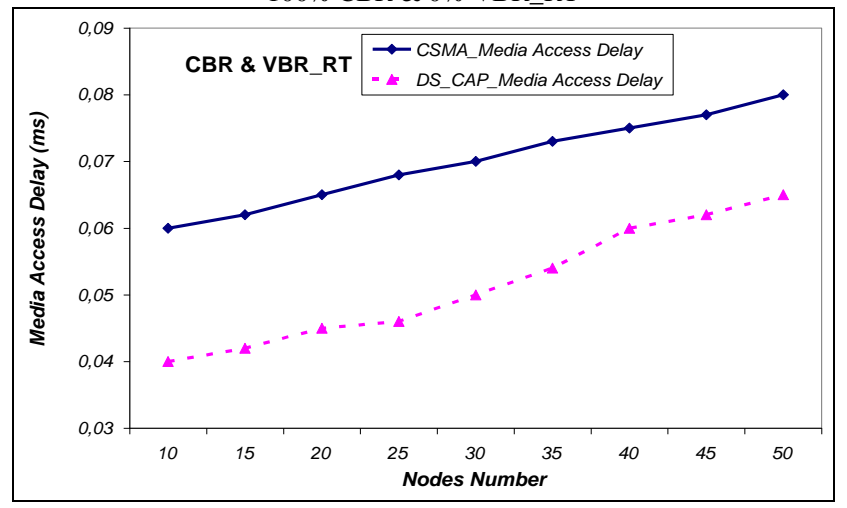

Figure 12. Media Access Delay evolution for applications $50 \%$ CBR \& 50\% VBR_RT

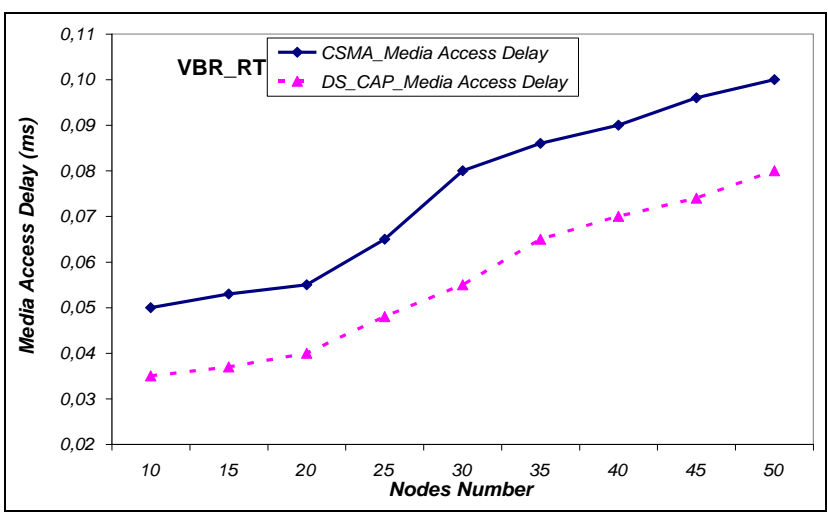

Figure 13. Media Access Delay evolution for applications 0\% CBR \& 100\% VBR_RT

\subsubsection{Simulation results for QSRR mechanism}

In this sub-section, we try to show that the performances provided by our resources reservation mechanism, QSRR, are always better than the ones provided by the CSMA protocol. Theses performances are expressed in terms of load.

In a general manner, we notice that the load increases with the increase of the number of nodes constituting the network, but the load provided by QSRR remains always lower than the one provided by CSMA, and this whatever the distribution of the applications and whatever the number of nodes constituting our network. The Figure 14, Figure 15 and Figure 16 represent the QSRR and CSMA load's evolution respectively for the application distribution 100\%CBR and 0\%VBR_RT, 50\%CBR and 50\%VBR_RT and finally 0\% CBR and 100\% VBR_RT.

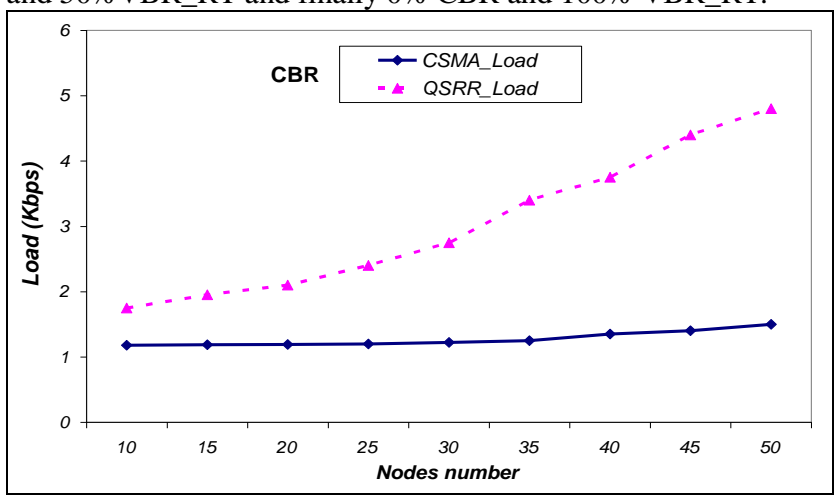

Figure 14. Load evolution for applications 100\% CBR \& 0\% VBR_RT

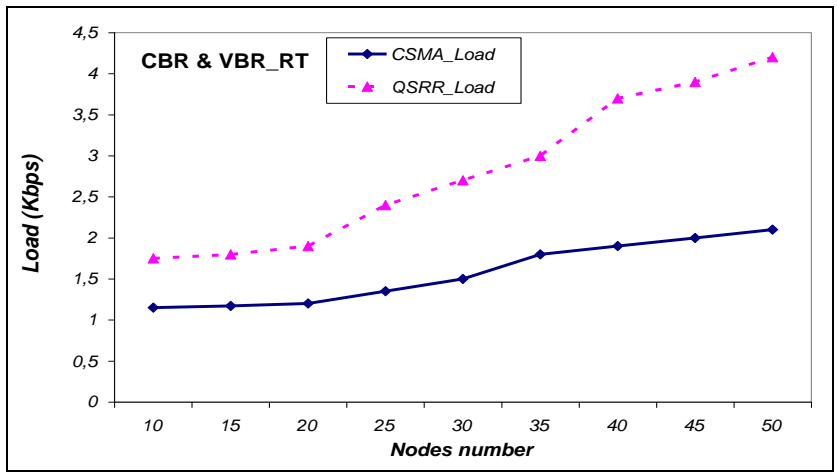

Figure 15. Load evolution for applications 50\% CBR \& 50\% VBR_RT 


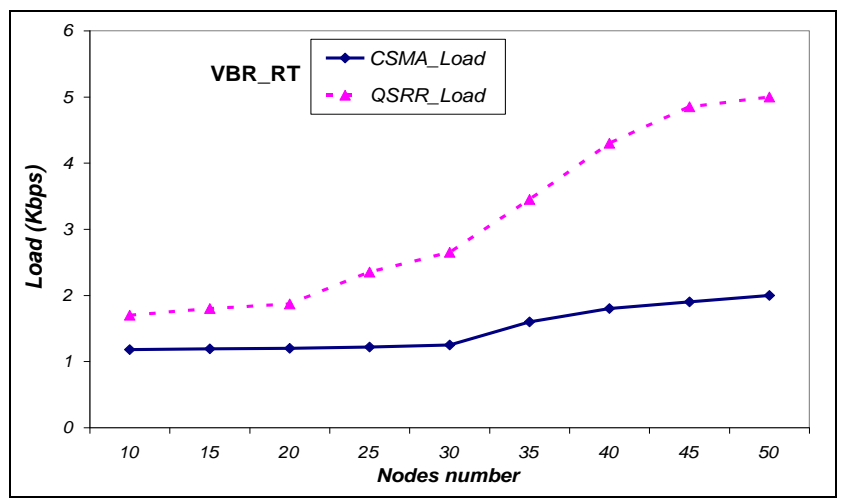

Figure 16. Load evolution for applications 0\%CBR\&100\%VBR_RT

\section{Conclusion}

The objective of our study was to elaborate a global architecture allowing us to provide preferential treatment for the real time applications by respecting their QoS requirements in term of delay and throughput.

The proposed routing protocol QoS oriented, QSDB, induces the introduction of additional control packets into network. We are conscious that this increase in the number of signaling packets in network will generate a rise of the use of resources and especially the bandwidth. However, the advantages brings to our network, justify its integration well. In fact, this protocol allows us to guarantee QoS to applications of network, to personalize and differentiate the provided service, to fulfill the requirements of various users, and, to optimize the use of network resources.

Then, we have proposed a collision management mechanism oriented QoS, called DS-CAP, which is adopted for introducing a service differentiation and an admission control oriented service for the AHNs users. The implementation of this mechanism provides a support access management based on the traffic priorities and allows the applications to clearly express their QoS requirements according to different metrics. These requirements are used to differentiate the appropriate treatment followed by the traffic. This novel mechanism is based on an adaptative Backoff traffic classes' differentiation, a service differentiation at the MAC and a traffic classes' differentiation where we suggest differentiating the policy of access to the channel according to the nature of flows.

In this paper, we also focused on a novel proposition of resources reservation mechanism of the traffic submitted to the network with regard to its QoS characteristics by defining a relation between the required constraints of the applications. Our QoS resources reservation method, QSRR, provides us reserving network path that have sufficient resources to satisfy the QoS requirements of all admitted connections. In this sense, for each flow on which we have provided some QoS guarantee constraints (in term of delay or throughput), QSRR will allocate some resources which will be exclusively for its use. While the resources reservation is based on the available bandwidth, we have use, in QSRR, an efficient method to obtain a relation between the required delay and the required bandwidth.
Finally, simulation analysis have been conducted and produced some performance evaluation results showing that the performances provided by all our proposed protocol and mechanisms are always better than the ones provided by existing mechanisms.

\section{REFERENCES}

[1] T. Imienlinski and B. R. Badrinath 'Mobile wireless computing: solutions and challenges in data management'. CACM, October 1994.

[2] M. S. Gast and O'Reilly, '802.11 Wireless networks: The definitive guide', 2002.

[3] G. Pujolle, 'Les Réseaux’, Troisième Edition, Eyrolles, 2002.

[4] M. Mirhakkak, N. Schult, et D. Thomson. 'Dynamic Quality of Service for Mobile Ad Hoc Networks', Dans IEEE MobiHoc 2000, Boston, Massachusets, USA, Août 2000.

[5] Marie-Ange Remiche, 'ELEC 379 : Réseaux II II', Belgique, 2005.

[6] Hassene Bouhouch and Sihem Guemara El Fatmi, "QoS Routing In Ad Hoc Networks by Integreting Activity in the OLSR Protocol" ICSNC, pp.1, Second International Conference on Systems and Networks Communications (ICSNC 2007), 2007

[7] Hassene Bouhouch and Sihem Guemara El Fatmi, " A Quality of Service Delay and Bandwidth-based Routing Protocol in Ad Hoc Networks", 2010 IEEE International Conference on Future Information technology (IEEE ICIFIT 2010), 2010.

[8] C. Sarr, C. Chaudet, G. Chelius and I. Guérin Lassous, "ABE : Un protocole de réservation de bande passante pour les réseaux ad hoc basés sur IEEE 802.11", Technical Report, INSA-Lyon, France, 2008.

[9] C. Sarr, C. Chaudet, G. Chelius and I. Guérin Lassous, 'ABE : Un protocole de réservation de bande passante pour les réseaux ad hoc basés sur IEEE 802.11', Technical Report, INSA-Lyon, France, 2008.

[10] N. Kettaf, H. Abouaissa, T. Vuduong and P. Lorenz, 'A Cross Layer Control On-demand Routing Protocol for QoS Applications', IJCSNS International Journal of Computer Science and Network Security, Vol 6, N 9B, September 2006.

[11] Hassene Bouhouch and Sihem Guemara El Fatmi, " Collision Management and Admission Control oriented Service Differentiation for Ad Hoc Netwoks", 2010 IEEE International Conference on Future Information technology (IEEE ICIFIT 2010), 2010.

[12] Hassene Bouhouch and Sihem Guemara El Fatmi, "Flexible Resources Reservation Scheme for Ad hoc Networks", 2010 IEEE International Conference on Information Theory and Information Security (IEEE ICITIS 2010), 2010. 